\title{
EXPLORING THE EFFECTS OF SOCIAL PREFERENCE, ECONOMIC DISPARITY, AND HETEROGENEOUS ENVIRONMENTS ON SEGREGATION*
}

\author{
NANCY RODRÍGUEZ ${ }^{\dagger}$ AND LENYA RYZHIK ${ }^{\ddagger}$
}

\begin{abstract}
It is believed that social preference, economic disparity, and heterogeneous environments are mechanisms that lead to segregation. However, it is difficult to unravel the exact role of each mechanism in a complex social system. We introduce a versatile, simple and intuitive particleinteraction model that allows one to separate the effect of each of these factors. As the population size and number of groups with different economic status approach infinity, we derive various macroscopic models for the population density. Through the analysis of the continuous limits, we conclude that, within this range of models, social preference is a necessary but not always sufficient mechanism for segregation. On the other hand, when combined with the environment and economic disparity (which on the their own also do not cause segregation), social preference does enhance segregation.
\end{abstract}

Key words. Parabolic system, degenerate diffusion, non-local interactions, particle-interaction model.

AMS subject classifications. 35K55, 35K57,35K65,35Q92.

\section{Introduction}

The separation along the lines of age, race, religious affiliation, and social status, to name a few, has been a ubiquitous phenomenon throughout time and cultures $[3,5,24]$. It appears that social segregation is a fundamental characteristic of human nature, and yet there is still much we do not understand of the mechanisms behind it, in spite of the vast literature on this subject (see, for example, [10,11, 19,23,24,27] and references within). Much of this research was sparked by the pioneering work of Shelling in [24], which made the case that social preference alone facilitates segregation by studying simple mathematical models. Modeling social preferences is generally difficult, as they vary dramatically across cultures, generations, and even neighborhoods within a city. Although there is a large literature in this area, the results are often inconclusive [2]. For example, some research shows that misery likes company, in other words, people like to be around people who are less well-off than themselves [16]. On the other hand, it is typical that residential areas are, generally, segregated along income lines [8]. This motivates the need to go beyond social preferences and consider economic disparity, along with heterogeneous environments, where certain dwelling locations are more desirable than others, as reasons for social segregation. It is clear that the choice of where people live is highly influenced by location, housing density, reputation of neighborhoods, amenities, and security $[9,12,26]$, but is limited by their economic power. The recent work of Gauvin and collaborators in [11] provides a mathematical framework for this phenomenon. The agent-based model considered in this reference involves individuals heterogeneous in their ability to pay for a certain residence, and includes interactions between potential sellers and buyers. Through the use of numerical and linear stability analysis the authors conclude that social segregation was possible only if the social preference to be near people of similar or higher income was sufficiently strong. This

\footnotetext{
* Received: April 21, 2014; accepted (in revised form): February 16, 2016. Communicated by Lorenzo Pareschi.

${ }^{\dagger}$ UNC Chapel Hill Department of Mathematics, Phillips Hall Chapel Hill, North Carolina 27599, USA (nrod@unc.edu).

${ }^{\ddagger}$ Stanford University, Department of Mathematics, Building 380, Sloan Hall Stanford, California 94305, USA (ryzhik@math.stanford.edu).
} 
model included market matching and the complicated dynamics prevented the authors from deriving a continuous model.

Here, we introduce a basic (intentionally bordering on simplistic) interactingparticle model that can take into account different social factors, as well as provide the flexibility to model the environment, such as city amenities, educational systems, public transportation, and highways, but is amenable to mathematical analysis. Similar interacting-particle systems are widely used in physics (see, for example, [17]), and have recently been popularized to model aggregation $[6,20,21,25]$.

We focus on three basic mechanisms believed to play a role in segregation: social preference, economic disparity, and a heterogeneous environment. The dynamics involves individuals of variable socio-economic status who navigate through a prescribed environment. The economic status is accounted for by the ease with which each individual navigates the environment- their mobility. In terms of social preference, we assume that individuals favor being surrounded by others with similar mobility. Hence, the role of mobility is two-fold: (1) it measures the ease with which a certain economic class can navigate an environment, and (2) it provides a mechanism of segregation through social preference.

While individual behavior is important, certain macroscopic patterns arise when we observe the bulk behavior of the system. To study them, we derive the continuous limit of the interacting-particle model. We first consider the case when the mobility is discrete, which is a crude way to describe the interaction between various social groups. This leads to a system of partial differential equations of the Keller-Segel type with repulsion (see, for example, [7] and references therein for other problems where such equations arise)). Next, we allow the mobility to be continuous, leading to a reactionadvection-diffusion equation in $\mathbb{R}^{d+1}$ where $d$-dimensions come from the physical space and time, and the additional dimension represents the mobility space. The rigorous justification of the continuous models is a technical and non-trivial issue beyond the scope of this paper.

A clear benefit of the continuous model is the ability to carry out the mathematical analysis of its qualitative behavior. We observe that in many of the macroscopic models the social preference alone does not lead to social segregation. In particular, if the initial distribution is socially diverse, it will maintain its social diversity. On the other hand, mobility and the environment enhance segregation, but only when some social preference is present. Of course, the present work only touches the tip of the iceberg, as there are many generalizations that need to be made in order to move toward a realistic mathematical understanding of social segregation.

Outline: We summarize the results in Subsection 1.1. In Section 2 we introduce the interacting-particle system for two groups with distinct mobility and formally derive various systems of PDEs. Section 3 is devoted to the proofs of the main results (Theorem 1.3, Theorem 1.4 and Theorem 1.10). In Section 4 we introduce the interactingparticle model for groups with continuous mobility and derive the formal PDE for this system. In Section 5 we discuss and illustrate some numerical experiments.

1.1. Models and results. We now introduce the models and state the corresponding results, postponing the derivations of the models until later. We consider two cases: (1) mobilities are discrete - the population includes individuals that are either disadvantaged or affluent - this setting could be easily modified to include any finite number of groups, and (2) a continuous range of mobilities - this describes the more realistic continuous spectrum of socio-economic classes in the population. 
1.1.1. Background and notation. We assume that interactions between individuals are governed by a prescribed interaction potential. A system with $N$ individuals whose interactions are governed by a non-negative potential $V_{N}(x) \geq 0, x \in \mathbb{R}^{d}$, leads to the evolution equations

$$
\frac{d}{d t} x_{N}^{k}(t)=\frac{1}{N} \sum_{j=1, j \neq k}^{N} \nabla V_{N}\left(x_{N}^{k}(t)-x_{N}^{j}(t)\right) \text { for } k=1, \ldots, N .
$$

In (1.1), $x_{N}^{k}(t)$ represents the physical position of individual $k$ at time $t$. As the number of individuals increases, the strength and range of interaction can vary and we make the typical assumption that the potential $V_{N}(x)$ is a rescaled version of a fixed potential $V(x)$ :

$$
V_{N}(x)=N^{\beta} V\left(N^{\beta / d} x\right) \text { for } \beta \in[0,1] .
$$

In what follows, we denote by $\Omega$ a bounded subset of $\mathbb{R}^{d}$ or all of $\mathbb{R}^{d}$. Also, for any $f \in C_{0}^{2}\left(\mathbb{R}^{d}\right)$ and probability measure $\mu$ we denote

$$
\langle\mu, f\rangle=\int_{\Omega} f d \mu(x) .
$$

The mass of a function $h(x)$ will be denoted by

$$
\int_{\Omega} h(x) d x=M[h] .
$$

We use $*$ to denote the standard convolution:

$$
K * u(x)=\int_{\Omega} K(x-y) u(y) d y .
$$

For any function $\rho(x)$ we denote by $\rho^{\#}(x)$ its symmetric non-increasing rearrangement of $\rho(x)$. We keep in mind that

$$
\left\|\rho^{\#}(x)\right\|_{p}=\|\rho(x)\|_{p} \text { for } p \in[1, \infty)
$$

and that for $G(x)$ symmetric and non-increasing

$$
\int_{\mathbb{R}^{2}} G(x-y) h(x) g(y) d x d y \leq \int_{\mathbb{R}^{2}} G(x-y) h^{\#}(x) g^{\#}(y) d x d y,
$$

see for example [15].

1.1.2. Discrete economic status. Let $u(x, t), v(x, t): \Omega \times[0, \infty) \rightarrow[0, \infty)$ represent the densities of the groups with the mobilities $\Gamma_{1} \gg \Gamma_{2}$, respectively. The most general systems of partial differential equations we obtain as the macroscopic limits of the interacting-particle system (see (2.2)) are, in the non-local case:

$$
\begin{aligned}
& u_{t}=\sigma \Delta u+\eta \Delta u^{2}+\nabla \cdot(u \nabla G *(v-u))+\Gamma_{1} \nabla \cdot(u \nabla A(x)) \text { in } \Omega, \\
& v_{t}=\sigma \Delta v+\eta \Delta v^{2}+\nabla \cdot(v \nabla G *(u-v))+\Gamma_{2} \nabla \cdot(v \nabla A(x)) \text { in } \Omega, \\
& u(x, 0)=u_{0}(x) \geq 0 \text { and } v(x, 0)=v_{0}(x) \geq 0 \text { for } t=0, \\
& \left(\sigma \nabla u+\eta \nabla u^{2}+u \nabla G *(v-u)+\Gamma_{1} \nabla A(x)\right) \cdot n=0 \text { on } \partial \Omega, \\
& \left(\sigma \nabla v+\eta \nabla v^{2}+v \nabla G *(u-v)+\Gamma_{2} \nabla A(x)\right) \cdot n=0 \text { on } \partial \Omega,
\end{aligned}
$$


where $\eta, \sigma \geq 0$ are the diffusivities (linear and nonlinear, respectively) and $n$ is the outward normal derivative at the boundary of $\Omega$; and in the local case:

$$
\begin{aligned}
& u_{t}=\sigma \Delta u+\lambda_{1} \Delta u^{2}+\lambda_{2} \nabla \cdot(u \nabla v)+\Gamma_{1} \nabla \cdot(u \nabla A(x)) \text { in } \Omega, \\
& v_{t}=\sigma \Delta v+\lambda_{1} \Delta v^{2}+\lambda_{2} \nabla \cdot(v \nabla u)+\Gamma_{2} \nabla \cdot(v \nabla A(x)) \text { in } \Omega, \\
& u(x, 0)=u_{0}(x) \geq 0 \text { and } v(x, 0)=v_{0}(x) \geq 0 \text { for } t=0, \\
& \left(\sigma \nabla u+\lambda_{1} \nabla u^{2}+\lambda_{2} u \nabla v+\Gamma_{1} u \nabla A(x)\right) \cdot n=0 \text { on } \partial \Omega, \\
& \left(\sigma \nabla v+\lambda_{1} \nabla v^{2}+\lambda_{2} v \nabla u+\Gamma_{2} v \nabla A(x)\right) \cdot n=0 \text { on } \partial \Omega,
\end{aligned}
$$

with $\lambda_{1}, \lambda_{2}>0$. The derivation of these systems can be found in Section 2. For the applications of interest, it is suitable to consider non-negative initial data $u_{0}(x)$ and $v_{0}(x)$. The no-flux boundary conditions imply that the total number of individuals in the city is preserved in time. The derivation of these systems is quite standard. We present it in some detail in Section 2, in order to differentiate the regimes that lead to the local or non-local interaction terms.

On the non-local model. The two diffusive terms in (1.5a) and (1.5b) are ofa different origin. The linear diffusion comes from the unpredictable human behavior (modeled as random noise), and the nonlinear diffusion comes from need of individuals to have some personal space (leading to an overcrowding effect). The convolution term models the long-range attraction to the individuals of the same group and long-range repulsion from the individuals in the other group, with the interaction potential $G(x)$. The scalar field $A(x)$ describes the spatially heterogeneous environment, and the population densities are advected by the gradient of $A(x)$, with the velocity being proportional to $\Gamma_{1}$ for $u(x, t)$ and $\Gamma_{2}$ for $v(x, t)$. The assumption that $\Gamma_{1} \gg \Gamma_{2}$ reflects the economic disparity between the populations. System (1.5) preserves mass and non-negativity, that is

$$
\int_{\Omega} u(x, t) d x=M\left[u_{0}(x)\right] \text { and } \int_{\Omega} v(x, t) d x=M\left[v_{0}(x)\right]
$$

and $u(x, t), v(x, t) \geq 0$ for all $x \in \Omega$ and $t>0$. This system is, formally, the gradient flow of the free energy

$$
\mathcal{F}(t):=\mathcal{E}(t)+\mathcal{W}(t)+\mathcal{S}(t)+\mathcal{L}(t)
$$

Here, the entropy, which comes from the two dispersal mechanisms, is

$$
\mathcal{E}(t):=\int_{\Omega} \eta\left(u^{2}+v^{2}\right)+\sigma(u \log u+v \log v) d x
$$

the interaction energy, which comes from the long-range attraction for the intra-group interactions, is

$$
\mathcal{W}(t):=-\frac{1}{2} \int_{\Omega} \int_{\Omega} G(x-y)(u(x, t) u(y, t)+v(x, t) v(y, t)) d x d y,
$$

the segregation energy, which describes the long-range repulsion for inter-group interactions, is

$$
\mathcal{S}(t):=\int_{\Omega} \int_{\Omega} u(x, t) G(x-y) v(y, t) d x d y
$$


and the environment energy, which comes from the environment landscape, is

$$
\mathcal{L}(t):=\int_{\Omega} A(x)\left(\Gamma_{1} u(x, t)+\Gamma_{2} v(x, t)\right) d x .
$$

The segregation energy, $\mathcal{S}(t)$, provides an explicit measure of how segregated the system is. A state is completely segregated if

$$
\int_{\Omega} \int_{\Omega} u(x, t) G(x-y) v(y, t) d x d y=0
$$

that is, if the two groups do not interact at all, minimizing the segregation energy. The first observation is that (1.5) dissipates the total energy.

Proposition 1.1 (Formal energy dissipation). Let $u(x, t)$ and $v(x, t)$ be solutions to (1.5) then

$$
\mathcal{F}(t)+\int_{0}^{t} \mathcal{D}(s) d s \leq \mathcal{F}(0), \forall t>0,
$$

where the energy dissipation is

$$
\begin{aligned}
\mathcal{D}(s)= & \int_{\Omega} u\left|\nabla\left(2 \eta u+\sigma \log u+G *(v-u)+\Gamma_{1} A(x)\right)\right|^{2} d x \\
& +\int_{\Omega} v\left|\nabla\left(2 \eta v+\sigma \log v+G *(u-v)+\Gamma_{2} A(x)\right)\right|^{2} d x .
\end{aligned}
$$

The computation leading to (1.10) is standard (see, for example, [4]). To analyze the different roles of social preference, the environment, and economic disparity we first consider the case when dispersal is due only to Brownian motion, i.e., $\eta=0$. Another simple observation is that if economic disparity is not included in the model then a population, which is initially socially diverse, will remain diverse for all time.

Proposition 1.2 (Lack of segregation). Let $\sigma>0, \eta=0$, and $\Gamma_{1}=\Gamma_{2}=0$. If $u(x, t)$ and $v(x, t)$ are solutions to (1.5) with initial conditions $u_{0}(x)=v_{0}(x)$ then $u(x, t)=v(x, t)$ for all $(x, t) \in \Omega \times(0, \infty)$.

More can be said when diffusion dominates the system: linearizing (1.5), with $\sigma>0$, $\eta=0$, and $\Gamma_{1}=\Gamma_{2}=0$, around the states $u \equiv v \equiv 1$ gives the linear system

$$
\begin{aligned}
& u_{t}=\sigma \Delta u+\Delta G *(v-u), \\
& v_{t}=\sigma \Delta v+\Delta G *(u-v),
\end{aligned}
$$

which is linearly stable if

$$
\sigma>2 \hat{G}(\xi)
$$

for all $\xi>0$, where $\hat{G}$ is the Fourier transform of $G$. Thus, we expect that, when (1.13) is satisfied, the "perfectly mixed" state $u \equiv v \equiv 1$ is non-linearly stable, so that there will be social diversity in the long run, rather than segregation. Here is a result in this direction.

TheOREM 1.3 (Preservation of social diversity). Let $u(x, t), v(x, t)$ be solutions to system (1.5) with $\sigma, \eta \geq 0, \Gamma_{1}=\Gamma_{2}=0$. Furthermore, assume that the initial conditions $u_{0}(x)$ and $v_{0}(x)$ satisfy

$$
M\left[u_{0}(x)\right]=M\left[v_{0}(x)\right]=M
$$


Then, for $\sigma>C(|\Omega|, G)$ sufficiently large the following estimate holds

$$
\|u-v\|_{L^{2}}^{2}+\|u+v-\bar{s}\|_{L^{2}}^{2} \leq\left(\left\|u_{0}(x)-v_{0}(x)\right\|_{L^{2}}^{2}+\left\|u_{0}(x)+v_{0}(x)-\bar{s}\right\|_{L^{2}}^{2}\right) e^{-C t},
$$

where $C\left(\sigma,\|\Delta G\|_{L^{\infty}(\Omega)}, M\right)$ and

$$
\bar{s}:=\frac{1}{|\Omega|} \int_{\Omega}(u+v) d x=\frac{2 M}{|\Omega|} .
$$

Thus, in the long time limit, the population density of affluent individuals is close to that of the disadvantaged individuals throughout the city. On the other hand, when (1.13) is violated we expect (at least, partially) segregated states in the long time limit.

Condition (1.14) is not necessary and is solely made to simplify the statement and the proof of the theorem. In general, if

$$
M\left[u_{0}(x)\right]=M_{u} \quad \text { and } \quad M\left[v_{0}(x)\right]=M_{v},
$$

then the following estimate holds,

$$
\left\|u-v-\bar{s}_{1}\right\|_{L^{2}}^{2}+\|u+v-\bar{s}\|_{L^{2}}^{2} \leq\left(\left\|u_{0}(x)-v_{0}(x)-\bar{s}_{1}\right\|_{L^{2}}^{2}+\left\|u_{0}(x)+v_{0}(x)-\bar{s}\right\|_{L^{2}}^{2}\right) e^{-C t},
$$

with

$$
\bar{s}_{1}=\frac{1}{|\Omega|} \int_{\Omega}(u-v) d x
$$

The next observation is that in an environment where the resources or amenities in a city are non-uniform, such as in mono-centric cities, disparity in mobility leads to segregation: if $\Gamma_{1} \gg \Gamma_{2}$, then $u(x, t)$ will be concentrated in areas where $A(x)$ is large and $v(x, t)$ will be concentrated in areas where $u(x, t)$ is low. We observe this behavior numerically, and prove a corresponding result for the non-local system with potentials that are close to the delta kernel: given a potential $G(x)$, set

$$
G_{\delta}(x):=\frac{1}{\delta^{d}} G\left(\frac{1}{\delta} x\right)
$$

THEOREM 1.4 (Segregated steady state for non-local system). Assume that $A(x) \in$ $C^{1}(\Omega), G(x) \in L^{1}\left(\mathbb{R}^{d}\right), \eta=0$, and $u_{0}(x), v_{0}(x) \in L^{1}(\Omega)$. There exists $\delta_{0}>0$ such that for $\delta<\delta_{0}$ there exist constants, $c_{1}, c_{2}>0$ and continuous functions $u_{\delta}(x)$ and $v_{\delta}(x)$ which satisfy

$$
\begin{aligned}
& u(x)=c_{1} \exp \left\{-\frac{1}{\sigma}\left(\Gamma_{1} A(x)+G_{\delta} *(v(x)-u(x))\right\}\right. \\
& v(x)=c_{2} \exp \left\{-\frac{1}{\sigma}\left(\Gamma_{2} A(x)+G_{\delta} *(u(x)-v(x))\right\}\right.
\end{aligned}
$$

and are steady-state solutions of system (1.5). Furthermore, the following hold:

$$
\int_{\Omega} u_{\delta}(x) d x=M\left[u_{0}(x)\right] \quad \text { and } \quad \int_{\Omega} v_{\delta}(x) d x=M\left[v_{0}(x)\right] .
$$


From (1.17) we observe that social preference is necessary for segregation but not sufficient. Indeed, without the social preference, when $G_{\delta}=0$, the ground states would have the form:

$$
\begin{aligned}
& u(x)=c_{1} \exp \left\{-\frac{\Gamma_{1}}{\sigma} A(x)\right\}, \\
& v(x)=c_{2} \exp \left\{-\frac{\Gamma_{2}}{\sigma} A(x)\right\},
\end{aligned}
$$

and would have qualitatively similar profiles.

The proof of Theorem 1.4 relies on the assumption that the dispersal is due only to random noise. The steady-state solutions in $\mathbb{R}^{d}$ when the dispersal includes the overcrowding effect, are the critical points of the energy functional (1.8) with $\eta>0$. As the parabolic system (1.5) conserves mass, it is reasonable to look for minimizers in the set

$$
\mathcal{Y}_{M_{u}, M_{v}}:=\left\{(u, v) \in\left(L_{+}^{1}\left(\mathbb{R}^{d}\right) \times L_{+}^{1}\left(\mathbb{R}^{d}\right)\right) \cap\left(L^{2}\left(\mathbb{R}^{d}\right) \times L^{2}\left(\mathbb{R}^{d}\right)\right):\|u\|_{1}=M_{u},\|v\|_{1}=M_{v}\right\},
$$

for $M_{u}, M_{v}>0$. The next result states that the energy minimizers in the set above are indeed steady state solutions to (1.5).

Proposition 1.5 (Stationary solutions via energy minimization). Let $(u, v) \in \mathcal{Y}_{M_{u}, M_{v}}$ be a minimizer of $\mathcal{F}[u, v]$, then $(u, v)$ satisfies

$$
\begin{aligned}
& \nabla(2 \eta u+G *(v-u))=0, \\
& \nabla(2 \eta v+G *(u-v))=0,
\end{aligned}
$$

a.e. in $x \in \mathbb{R}^{d}$.

Proposition 1.6 (Regularity of steady-states). Let $\left(u_{0}(x), v_{0}(x)\right) \in \mathcal{Y}_{M_{u}, M_{v}}$ then the solutions $(u(x, t), v(x, t))$ to $(1.5)$ with $\sigma=\Gamma_{1}=\Gamma_{2}=0$ satisfy

$$
\int_{\mathbb{R}} u|\nabla u|^{2}+v|\nabla v|^{2} d x<\infty, \quad \text { a.e. } t>0 .
$$

In particular, any steady-state solutions to (1.5) with $\sigma=\Gamma_{1}=\Gamma_{2}=0,(u, v)$, satisfies (1.20) and both $u$ and $v$ are $C^{2}$ on their support.

A formal analysis of (1.8) gives us hints at the behavior of solutions to system (1.5) and also to the existence of minimizers in $\mathcal{Y}_{M_{u}, M_{v}}$. The choice of interaction potential affects the system in two key ways: the strength of its pull at the origin and its decay as $|x| \rightarrow \infty$. The former provides a measure of the risk of finite-time blow-up of the solutions. The latter provides a pull of mass when it is trying to escape to infinity. In other words, if the pull is too weak (and we can additionally rule out finite-time blow up) we expect that no minimizers will exist.

In this work we only consider interaction potentials that guarantee that (1.5) is a diffusion dominated system. Consider what happens to the energy as we rescale $u(x)$ and $v(x)$ into delta functions, $u_{\lambda}=\lambda^{d} u(\lambda x)$ and $v_{\lambda}=\lambda^{d} v(\lambda x)$. We obtain the rescaled energy,

$$
\begin{aligned}
\mathcal{F}\left[u_{\lambda}, v_{\lambda}\right] & =\eta \lambda^{d}\left(\|u\|_{2}^{2}+\|v\|_{2}^{2}\right)-\frac{1}{2}\left(\int_{\mathbb{R}} G\left(\frac{x-y}{\lambda}\right)(u(x) u(y)+v(x) v(y)-2 u(x) v(y)) d x d y\right) \\
& \geq \lambda^{d} \eta\left(\|u\|_{2}^{2}+\|v\|_{2}^{2}\right)-\|G\|_{\infty}\left(M_{u}^{2}+M_{v}^{2}\right) .
\end{aligned}
$$


Taking the limit as $\lambda \rightarrow \infty$ gives $\mathcal{F}\left[u_{\lambda}, v_{\lambda}\right] \rightarrow \infty$. From this heuristic, we expect the solutions to exists globally and the natural question to ask then is if the solutions weak* converge to zero or if there exists a nontrivial ground state. Next, let us analyze what happens to the energy as the mass of a sequence in $\mathcal{Y}_{M_{u}, M_{v}}$ spreads out and the center of mass of $u(x)$ and $v(x)$ shift further away from each other. Take $(u(x), v(x)) \in \mathcal{Y}_{M_{u}, M_{v}}$ and consider the mass invariant scaling with a shift

$$
u_{\lambda}(x)=\lambda^{d} u\left(\lambda\left(x-x_{\lambda}\right)\right) \quad \text { and } \quad v_{\lambda}(x)=\lambda^{d} v\left(\lambda\left(x+x_{\lambda}\right)\right)
$$

where $\left\{x_{\lambda}\right\}_{\lambda>0}$ is a sequence in $\mathbb{R}^{d}$ with $\lim _{\lambda \rightarrow 0}\left|x_{\lambda}\right|=\infty$. This time we are interested in what happens when $\lambda \rightarrow 0$,

$$
\begin{aligned}
\mathcal{F}\left[u_{\lambda}, v_{\lambda}\right]= & \eta \lambda^{d}\left(\|u\|_{2}^{2}+\|v\|_{2}^{2}\right)-\frac{\lambda^{d}}{2} \int_{0}^{1} \int_{\mathbb{R}^{d}} \frac{1}{\lambda^{d}} G\left(\frac{x-y}{\lambda}\right)(u(x) u(y)+v(x) v(y)) d x d y \\
& +\lambda^{d} \int_{0}^{1} \int_{\mathbb{R}^{d}} \frac{1}{\lambda^{d}} G\left(\frac{x-y}{\lambda}+2 x_{\lambda}\right) u(x) v(y) d x d y
\end{aligned}
$$

Note that as $\lambda \rightarrow 0$ the second term scales as $\frac{\lambda^{d}}{2}\left\|G_{1}\right\|_{1}\left(\|u\|_{2}^{2}+\|v\|_{2}^{2}\right)$. Thus, if $\left\|G_{1}\right\|_{1}<2 \eta$ the energy is always non-negative and we can find a suitable subsequence of $\mathcal{Y}_{M_{u}, M_{v}}$ that weak* converges to zero. Thus, we conclude

$$
\inf _{u, v \in \mathcal{Y}_{M_{u}, M_{v}}} \mathcal{F}[u, v]:=I_{M_{u}, M_{v}} \leq 0 .
$$

Lemma 1.7 (Complete segregation). Any non-trivial minimizer of $\mathcal{F}[u, v],\left(u^{*}, v^{*}\right) \in$ $\mathcal{Y}_{M_{u}, M_{v}}$, must have disjoint supports. In particular, if $\operatorname{supp}(G) \subset B_{R}(0)$ then $\operatorname{dist}\left(\operatorname{supp}\left(u^{*}\right), \operatorname{supp}\left(v^{*}\right)\right) \geq 2 R$ and

$$
S\left[u^{*}, v^{*}\right]=0 .
$$

TheOREM 1.8 (Nonexistence of minimizers). Let $G \in L^{1}\left(\mathbb{R}^{d}\right), M_{u}, M_{v}>0$. If $\|G\|_{1}<2 \eta$ or $G$ has unbounded support then there are no minimizers of $\mathcal{F}[u, v]$ in $\mathcal{Y}_{M_{u}, M_{v}}$.

On a final note, making a rigorous connection between the interacting-particle model and the continuous system (1.5) requires the global well-posedness of (1.5). The global well-posedness result for weak-solutions, which is the best we can hope for when $\sigma=0$ due to the degenerate diffusion, is stated below. However, we leave the proof for a more technical paper in preparation.

TheOrem 1.9 (Global well-posedness). Let $\sigma=0$ and $G(x) \in C^{2}(\Omega) \cap W^{1,2}(\Omega)$ be admissible. Let $u_{0}(x), v_{0}(x) \in L^{\infty}(\Omega)$ be non-negative initial conditions. System $(1.5)$ has weak solutions $u(x, t), v(x, t) \in L^{\infty}(\Omega \times(0, T))$ for any $T>0$.

Note that when $\sigma>0$ the solutions are classical.

On the local model. As mentioned earlier, it is important to understand how the assumptions on the microscopic interactions affect the large population limit, which is one reason to consider the local model. We have the following version of Theorem 1.4 for the local system.

TheOREM 1.10 (Segregated steady state for the local system). Let $A(x) \in L^{\infty}(\Omega), \lambda_{1}=$ 0 , and $u_{0}(x), v_{0}(x) \in L^{1}(\Omega)$. There exist two positive constants, $c_{1}, c_{2}$, and continuous 
functions, $\bar{u}(x)$ and $\bar{v}(x)$, which are positive and satisfy

$$
\begin{aligned}
& \bar{u}(x)=c_{1} \exp \left\{-\frac{1}{\sigma}\left(\Gamma_{1} A(x)+\lambda_{2} \bar{v}(x)\right)\right\}, \\
& \bar{v}(x)=c_{2} \exp \left\{-\frac{1}{\sigma}\left(\Gamma_{2} A(x)+\lambda_{2} \bar{u}(x)\right)\right\},
\end{aligned}
$$

that are steady-state solutions of system (1.6). Additionally, the following holds:

$$
\int_{\Omega} \bar{u}(x) d x=M\left[u_{0}(x)\right] \quad \text { and } \quad \int_{\Omega} \bar{v}(x) d x=M\left[v_{0}(x)\right] .
$$

It is worth noting that the local and global well-posedness for (1.6) is still an open problem.

1.1.3. Continuous income spectrum. So far, we have assumed that the mobility is discrete. The models discussed above can be easily extended to a continuous mobility spectrum. Starting from the interacting-particle model, we not only take the limit as the number of individuals approach infinity, but also as the number of groups, each with a different mobility, approaches infinity. We will assume that the mobility $y$ is an independent variable, normalized so that $y \in[0,1]$. It is helpful to define a mobility threshold, $\kappa \in[0,1]$, as a parameter that measures the line between attraction and repulsion based on mobility. An individual with mobility $y_{1}$ is attracted to an individual with mobility $y_{2}$ if $\left|y_{1}-y_{2}\right| \leq \kappa$, while the individuals repulse each other if $\left|y_{1}-y_{2}\right|>\kappa$. The interactions can be governed, for example, by the potential $H(y) G(x)$, where

$$
H(y)=\kappa|y|-1
$$

determines the attractive or repulsive nature of the interaction, and $G(x)$ determines the strength of the interaction due to the spatial distance between the individuals. The population density $u(x, y, t)$ at location $x \in \mathbb{R}^{d}$, with mobility $y \in[0,1]$ at time $t$, satisfies the following partial differential equation

$$
\begin{aligned}
\partial_{t} u(x, y, t)= & \eta \nabla_{x} \cdot\left(u(x, y, t) \nabla_{x} \bar{u}(x, t)\right) \\
& \left.-\nabla_{x} \cdot\left(u(x, y, t) \nabla_{x}\left[\int_{0}^{1} \int_{\mathbb{R}^{d}} H(y-\tilde{y}) G(x-\tilde{x}) u(\tilde{x}, \tilde{y}, t)\right) d \tilde{x} d \tilde{y}+y A(x)\right]\right),
\end{aligned}
$$

where $\nabla_{x}$ denotes the spatial gradient and

$$
\bar{u}(x, t)=\int_{0}^{1} u(x, y, t) d y .
$$

Notice that the first term on the right-hand side of (1.24) is reminiscent of porousmedia diffusion. Indeed, the equation is advecting $u(x, y, t)$ down the gradients of $\bar{u}(x, t)$, which provides a measure for the total population density. Equation (1.24), with noflux boundary conditions, conserves mass and non-negativity and also rearranges the mobility.

1.1.4. Crime, safety, and economic disparity. As we have mentioned, one benefit of the models we introduce in this work is the freedom to explore the effects of heterogeneous environments. As an example, we consider a scalar field $A(x, t)$ which 
measures the probability that a criminal act will occur at location $x$ and time $t$. In this setting, we can explore various hypotheses related to the interconnection between crime hotspots, defined to be spatio-temporal areas of high density of criminal activity, and the local economic status of the population. One objective is to recreate the phenomena of the concentration of crime hotspots in low-income neighborhoods, a trend which has been observed recently $[13,14]$. It is important to note that crime is an extremely complex phenomena and it would be naive to identify the model we propose below as reality. In fact, we do not account for the complex issues that poverty can create, which may also play a role in the increase of crime, such as lack of employment and education. The goal is to identify possible mechanisms, which do not assume that poverty leads to crime, but can recreate the phenomenon of unusually high crime in extremely disadvantaged neighborhoods. This does not imply that such mechanisms are the cause of the high crime rates, but indicates that they are worth understanding. While the role the government plays with regards to crime is not exactly clear, we assume that its objective is to minimize the "fear of crime" [22]. Research done in this area points to the inability of individuals to rationally calculate the objective danger. For instance, certain subpopulations tend to be more fearful of crime than others (see [22] and references within).

Given a distribution of the population at time $t$ we consider an influence field, $I(x, t)$, which measures the economic power of a certain location at that time, and let $p(x, t)$ be the distribution of police resources at time $t$. The insecurity functional at time $t$, $U(x, t)$, depends on $I(x, t)$ and measures how unsafe the population feels. For example, under the assumption that people get used to crime, (people who live in a high-crime neighborhood are less phased by a single criminal activity whereas the insecurity of the people who live in a safe neighborhood will significantly increase with a single criminal activity), one may consider

$$
\begin{aligned}
& U(p(x, t))=\sqrt{\delta+\exp (-\alpha p(x, t)) I(x, t)} \text { or } \\
& U(p(x, t))=\sqrt{\delta+\exp (-\alpha p(x, t)) \exp (I(x, t))}
\end{aligned}
$$

where $\delta>0$ is a parameter that measures the minimum amount of insecurity and $\alpha>0$ measures the effectiveness of the police recourses. We assume that the police aims to minimize the functional

$$
F(p)=\int_{\Omega}\left[\varepsilon|\nabla p(x, t)|^{2}+U(p(x, t))\right] d x
$$

where the first term is a regularization to provide some smoothness. Taking into account the normalization due to a fixed total amount of police resources, the variational formulation for the distribution of the police resources, given the population income distribution $I(x, t)$ is:

$$
\begin{aligned}
& \min _{p \geq 0} F(p) \\
& \text { subject to } \int_{\Omega} p(x, t)=1 .
\end{aligned}
$$

Given the optimal use of resources, $p^{*}(x, t)$, which satisfies (1.28), we assume an inverse relationship between $A(x, t)$ and $p^{*}(x, t)$. For example,

$$
A(x, t)=\exp \left\{-\alpha p^{*}(x, t)\right\}
$$


From a prescribed distribution of the population we generate the influence field and given this field we then use a gradient decent scheme to solve (1.28). Numerically, we observe the development of "safe-havens," regions with very low values of $A(x, t)$, in ares where there is high economic power, refer to Figure 5.4b for an illustration.

\section{Discrete mobility model}

In this section, we consider discrete mobilities, corresponding to a discrete number of social classes.

2.1. Interacting particle model. We consider two distinct groups, of $N_{1,2}$ individuals, with the mobilities $\Gamma_{1} \gg 1, \Gamma_{2} \ll 1$, that exhibit different inter-group and intra-group interactions. The individual dynamics is governed by three rules:

(A1) overcrowding effect: although individuals from the same group are attracted in the long-range, the desire for some personal space leads to local (short-range) repulsion.

(A2) social preference segregation: individuals are attracted to those in their own group and repulsed from those in the other group, leading to long-range attraction between individuals of the same group and repulsion between individuals in different groups.

(A3) environment field $A(x)$ : individuals are attracted to either low or high values of the scalar field, $A(x)$, depending on what $A(x)$ represents. For example, if $A(x)$ represents the quality of the educational system then individuals have the preference to be in areas where $A(x)$ is high. On the other hand, if $A(x)$ represents the crime density then individuals have the preference to be in areas with small values of $A(x)$. The velocity of each individual is proportional to its mobility.

We assume that for the intra-group interactions the short-range repulsion is governed by the interaction potential $V_{N}(x)$ and the long-range mobility segregation is governed by the interaction potential $G_{N}(x)$, of the form

$$
V_{N}(x)=N^{\gamma_{v}} V_{1}\left(N^{\gamma_{v} / d} x\right) \text { and } G_{N}(x)=N^{\gamma_{g}} G_{1}\left(N^{\gamma_{g} / d} x\right),
$$

with $\gamma_{v}, \gamma_{g} \in[0,1)$, respectively. The parameters, $\gamma_{v}$ and $\gamma_{g}$ determine the strength and range of the interactions as the number of individuals in the system increases. Combining these effects, we obtain the following system of ODEs for the positions $x_{i}^{k}(t)$ with $i=1,2$ :

$$
\begin{aligned}
\frac{d}{d t} x_{i}^{k}(t)= & -\frac{1}{N_{i}} \sum_{j=1, j \neq k}^{N_{i}}\left(\nabla V_{N_{i}}\left(x_{i}^{k}(t)-x_{i}^{j}(t)\right)-\nabla G_{N_{i}}\left(x_{i}^{k}(t)-x_{i}^{j}(t)\right)\right) \\
& -\frac{1}{N_{l}} \sum_{j=1}^{N_{l}} \nabla G_{N_{l}}\left(x_{i}^{k}(t)-x_{l}^{j}(t)\right)-\Gamma_{i} \nabla A\left(x_{i}^{k}(t)\right),
\end{aligned}
$$

for $k=1, \ldots, N_{i}, l=1,2$, and $i \neq l$. The dynamics of both groups are identical except for the difference in mobility. This model can be easily extended to $n$ groups.

2.2. Continuum limit for the two-population model. In order to analyze the continuous limit (large $N_{1,2}$ ), we define the empirical measures

$$
X_{i}(t)=\frac{1}{N_{i}} \sum_{j=1}^{N_{i}} \delta_{x_{i}^{j}(t)} \quad \text { for } \quad i=1,2 .
$$


From a macroscopic perspective, we assume that there is a function $u_{i}(x, t) \in \mathbb{R}^{d} \times(0, \infty)$, which represent the density distribution of the two groups at time $t$ :

$$
\lim _{N_{i} \rightarrow \infty}\left\langle X_{i}(0), f\right\rangle=\left\langle u_{i 0}(\cdot), f\right\rangle \quad \text { and } \quad \lim _{N_{i} \rightarrow \infty}\left\langle X_{i}(t), f\right\rangle=\left\langle u_{i}(\cdot, t), f\right\rangle \quad \forall t>0,
$$

for $i=1,2$. Since the formal derivation of the continuous limit is standard and is similar to the derivation of (1.24) in Section 4, we omit the details but mention that the type of limiting equations that one obtains depends on the assumptions made on the scaling parameters. One obtains two types of continuous models. First, a non-local model comes about when $\gamma_{v} \in(0,1)$ and $\gamma_{g}=0$ :

$$
V_{N}(x)=N^{\gamma_{v}} V_{1}\left(N^{\gamma_{v} / d} x\right) \text { and } G_{N}(x)=G_{1}(x) .
$$

For simplicity, from now on we will denote $V=V_{1}$ and $G=G_{1}$. "Physically", this means that individuals within the same group are repulsed locally and attracted long-range, describing the balance of individuals' social nature and the need for personal space. On the other hand, individuals are repulsed at a long-range from individuals belonging to another group. The local equilibrium hypothesis states that locally the individuals are distributed in a uniform manner - the typical distance between individuals in group one close to a location $x$ is $\left(N_{1} u(x, t)\right)^{-1}$, and similarly for group two [20,21]. From these assumptions one obtains the weak form of system (1.5) with $\sigma=0, \eta=M[V] / 2$, and $\left(u_{1}, u_{2}\right)$ replaced by $(u, v)$. On the other hand, if we assume that both $\gamma_{v} \in(0,1)$ and $\gamma_{g} \in(0,1)$, then we obtain the local system of equations (1.6) with $\sigma=0$,

$$
\lambda_{1}=\frac{1}{2}(M[V]-M[G]) \quad \text { and } \quad \lambda_{2}=\frac{1}{2} M[G] .
$$

The mass of the attractive potential $G(x)$ (the segregation potential) relative to that of the repulsive potential $V(x)$ determines the sign of $\lambda_{1}$. For well-posedness of the system (1.6), one has to assume that diffusion overpowers aggregation so that $\lambda_{1}>0$.

2.3. Individual preference through random noise. Thus far, we have assumed that all individuals follow the exact same rules of interaction. In reality, however, individuals have personal preferences that do not necessarily follow the deterministic dynamics. We may account for personalized preferences through a random noise, in which case the position of the individuals

$$
\left\{x_{i}^{k}(t)\right\}_{t>0}, k=1, \ldots, N_{i} \text {, and } i=\{1,2\},
$$

is described as a stochastic process described by a stochastic differential equation

$$
d x_{i}^{k}(t)=\left[T\left(x_{1}(t), x_{2}(t)\right)+\Gamma_{i} A\left(x_{i}^{k}(t)\right)\right] d t+\sigma d W_{i}^{k}(t),
$$

for $k=1, \ldots, N_{i}$ and $i=\{1,2\}$. Here $W_{i}^{k}$ is a family of independent standard Wiener processes. The function $T$ includes all of the intra-group and inter-group interactions we have considered above. Following the formal derivations of [18], which is easily adapted to systems, we obtain the general models (1.5) and (1.6) (once again $\left(u_{1}, u_{2}\right)$ is replaced by $(u, v))$. We refer the interested reader to [18] for more details.

\section{Social diversity vs. segregation}

In this section, we explore whether a population preserves social diversity or moves toward a segregated state. 
Social diversity. Here, we prove Theorem 1.3.

Proof. (Theorem 1.3.) Let $u(x, t)$ and $v(x, t)$ be solutions to system (1.5) with $\Gamma_{1}=\Gamma_{2}=\eta=0$, and define

$$
w=u-v \quad \text { and } \quad s=u+v-\bar{s},
$$

where $\bar{s}$ is defined in (1.16). First, assume that $\eta=0$ :

$$
\begin{aligned}
w_{t} & =\sigma \Delta w-\nabla \cdot(s \nabla G * w)-\bar{s} \Delta G * w, \\
s_{t} & =\sigma \Delta s-\nabla \cdot(w \nabla G * w),
\end{aligned}
$$

with initial conditions

$$
w(x, 0)=u_{0}(x)-v_{0}(x) \text { and } s(x, 0)=u_{0}(x)+v_{0}(x)-\bar{s},
$$

and no-flux boundary conditions. Multiplying (3.1a) by $w(x, t)$ and (3.1b) by $s(x, t)$ we obtain the estimates

$$
\begin{aligned}
\frac{d}{d t}\left(\|w\|_{L^{2}(\Omega)}^{2}+\|s\|_{L^{2}(\Omega)}^{2}\right) & =-\sigma\left(\|\nabla w\|_{L^{2}}^{2}+\|\nabla s\|_{L^{2}}^{2}\right)+\int_{\Omega} s(\nabla G * w) \nabla w d x \\
& +\int_{\Omega} w(\nabla G * w) \nabla s d x-\int_{\Omega} \bar{s}(\Delta G * w) w d x:=-I_{1}+I_{2}+I_{3}+I_{4} .
\end{aligned}
$$

Note that both $w(x, t)$ and $s(x, t)$ have mean zero mass, the latter due to (1.14) and the former due to its definition. Therefore, we can apply Poincaré inequality to $I_{1}$ and obtain

$$
-I_{1}<-C_{p} \sigma\left(\|w\|_{L^{2}(\Omega)}^{2}+\|s\|_{L^{2}(\Omega)}^{2}\right) .
$$

Next, we integrate by parts the term $I_{2}$ and add it to $I_{3}$ to obtain

$$
I_{2}+I_{3}=-\int_{\Omega} s w(\Delta G * w) d x \leq \frac{1}{2}\|\Delta G\|_{L^{\infty}(\Omega)}\|w\|_{L^{1}(\Omega)}\left(\|w\|_{L^{2}(\Omega)}^{2}+\|s\|_{L^{2}(\Omega)}^{2}\right) .
$$

The final bound seen in (3.3) is obtained by the use of Young's inequality for convolutions and the Cauchy-Schwarz inequality. For the last term we obtain

$$
I_{4} \leq \bar{s}\|\Delta G\|_{L^{1}(\Omega)}\|w\|_{L^{2}(\Omega)}^{2} .
$$

Thus, combining (3.2)-(3.4) we obtain

$$
\begin{aligned}
\frac{d}{d t}\left(\|w\|_{L^{2}(\Omega)}^{2}+\|s\|_{L^{2}(\Omega)}^{2}\right) \leq & \left(-C_{p} \sigma+\frac{1}{2}\|\Delta G\|_{L^{\infty}(\Omega)}\|w\|_{L^{1}(\Omega)}+\bar{s}\|\Delta G\|_{L_{1}(\Omega)}\right)\|w\|_{L^{2}}^{2} \\
& +\left(-C_{p} \sigma+\frac{1}{2}\|\Delta G\|_{L^{\infty}(\Omega)}\|w\|_{L^{1}(\Omega)}\right)\|s\|_{L^{2}(\Omega)}^{2} .
\end{aligned}
$$

Choosing $\sigma>\frac{1}{C_{p}}\left(\frac{1}{2}\left\|\left.\Delta G\right|_{L^{\infty}(\Omega)}\right\| w\left\|_{L^{1}(\Omega)}+\bar{s}\right\| \Delta G \|_{L_{1}(\Omega)}\right)$ we obtain the differential inequality:

$$
\frac{d}{d t} y \leq-C y, \quad y(0)=\left\|u_{0}(x)-v_{0}(x)\right\|_{L^{2}}^{2}+\left\|u_{0}(x)+v_{0}(x)-\bar{s}\right\|_{L^{2}}^{2},
$$

where $y=\|w\|_{L^{2}}^{2}+\|s\|_{L^{2}}^{2}$ and $C=C(\sigma, \bar{s}, G, M)$. Integrating (3.5), we obtain estimate $(1.15)$. 
Segregation due to mobility disparity and environment: the nonlocal case. This section is devoted to the proof of Theorem 1.4. The first step is to establish that (1.17) has a non-trivial solution $\left(u_{0}, v_{0}\right) \in C^{1}(\Omega) \times C^{1}(\Omega)$ for any $c_{1}, c_{2}>0$ when $\delta=0$. Second, we invoke the implicit function theorem to show that the same holds in a small neighborhood of $\delta=0$. Finally, we prove that there are constants $c_{1}, c_{2}>0$ such that $(1.17 \mathrm{c})$ holds.

Proof. Without loss of generality assume that $\sigma=1$.

Step 1: Define $f_{i}(x)=\exp \left\{-\Gamma_{i} A(x)\right\}$ for $i=1,2$, and $g(x)=f_{1}(x) f_{2}(x)$. Note that

$$
u(x) v(x)=c_{1} c_{2} g(x),
$$

and since $c_{1} c_{2} g(x)>0$ we can express $v$ in terms of $u$

$$
v(x)=\frac{c_{1} c_{2} g(x)}{u(x)} .
$$

This reduces the problem to solving the following fixed point problem

$$
u(x)=c_{1} f_{1}(x) e^{u(x)-\frac{c_{1} c_{2} g(x)}{u(x)}} .
$$

For $\bar{x} \in \Omega$ fixed this is equivalent to solving $u=a e^{u-\frac{b}{u}}$ where $a$ and $b$ are positive constants, which always has a non-trivial solution as

$$
\lim _{u \rightarrow 0^{+}} f^{\prime \prime}(u)=0
$$

where we define $f(u):=a e^{u-\frac{b}{u}}$. Repeating this process for all $x \in \Omega$ gives a solution $\left(u_{0}(x), v_{0}(x)\right)$ to (1.17). Moreover, $u_{0}$ and $v_{0}$ inherit the regularity of $A(x)$ and there exist $K, \epsilon_{1}>0$ such that $\epsilon_{1}<u_{0}(x), v_{0}(x)<K$.

Step 2: Let $F(\delta, u):[0, \infty) \times C^{1}(\Omega) \rightarrow C^{1}(\Omega)$ defined by

$$
F(\delta, u):=u(x)-c_{1} f_{1}(x) \exp \left\{G_{\delta} *\left(u(x)-\frac{c_{1} c_{2} g(x)}{u(x)}\right)\right\} .
$$

Note that $F$ is a $C^{1}$ map, $F\left(0, u_{0}\right)=0$ and $D_{u} F\left(0, u_{0}\right) v: C^{1}(\Omega) \rightarrow C^{1}(\Omega)$ defined by

$$
D_{u} F\left(0, u_{0}\right) v=h(x) v,
$$

where $h(x)<\infty$ and strictly bounded from 0 , is an isomorphism. Then the implicit function theorem defines a unique mapping $\left(\delta, u_{\delta}\right)$ for $\delta$ near 0 and $u_{\delta}$ near $u_{0}$ such that $F\left(\delta, u_{\delta}\right)=0$.

Step 3: In the previous step we proved the existence of $u_{\delta}(x)$ and $v_{\delta}(x)$ for any positive and finite constants $c_{1}$ and $c_{2}$. Next we prove the claim that for any given $0<M_{1}, M_{2}<\infty$ there are corresponding $c_{1}$ and $c_{2}$ that allow $u_{\delta}(x)$ and $v_{\delta}(x)$ to satisfy $(1.17 \mathrm{c})$. Recall that we want

$$
\int_{\Omega} v_{\delta}(x) d x=c_{1} c_{2} \int_{\Omega} \frac{g(x)}{u_{\delta}(x)} d x=M_{2}
$$

which gives that $c_{2}=\frac{M_{2}}{c_{1} \int_{\Omega} \frac{g(x)}{u_{\delta}(x)} d x}$. Now, consider the mass of $u_{\delta}(x)$

$$
\int_{\Omega} u_{\delta}(x) d x=c_{1} \int_{\Omega} f_{1}(x) \exp \left\{G *\left(u_{\delta}(x)-\frac{M_{2}}{\int_{\Omega} \frac{g(y)}{u_{\delta}(y)} d y} \frac{g(x)}{u_{\delta}(x)}\right)\right\} d x \text {. }
$$


Again, when $c_{1}=0$ we see that $M\left[u_{\delta}(x)\right]=0$. In addition, we have the following lower bound for the mass of $u_{\delta}(x)$ :

$$
\begin{aligned}
\int_{\Omega} u_{\delta}(x) d x & \geq c_{1} \int_{\Omega} f_{1}(x) \exp \left\{-\frac{M_{2}}{\int_{\Omega} \frac{g(y)}{u_{\delta}(y)} d y} \int G(x-y) \frac{g(y)}{u_{\delta}(y)} d y\right\} d x \\
& \geq c_{1} e^{-\Gamma_{1} A_{\max }} \exp \left\{-M_{2}\|G\|_{L^{\infty}}\right\} .
\end{aligned}
$$

As before, this implies that we can choose $c_{1}$ sufficiently large such that $M\left[u_{\delta}(x)\right]>M_{1}$. By continuity, we conclude the proof.

Energy minimizers. Here, we look at the energy minimizers of (1.8) and their connection to the steady-state solutions to system (1.5). We begin with the proof of Lemma 1.7.

Proof. (Lemma 1.7.) Suppose there exists $\left(u^{*}, v^{*}\right) \in \mathcal{Y}_{M_{u}, M_{v}}$ with

$$
\operatorname{supp}\left(u^{*}\right) \cap \operatorname{supp}\left(v^{*}\right) \neq \emptyset,
$$

such that $F\left[u^{*}, v^{*}\right]=I_{M_{u}, M_{v}}$. By (3.7) it must be the case that

$$
\mathcal{S}\left(u^{*}, v^{*}\right)>0 \text {. }
$$

Let $u^{\#}(x)$ be the symmetric decreasing rearrangement of $u(x)$ (similarly for $v(x)$ ) and consider the sequence

$$
u_{n}(x):=u^{\#}\left(x+x_{n}\right) \text { and } v_{n}(x):=v^{\#}\left(x-x_{n}\right),
$$

for $\left\{x_{n}\right\}$ a sequence in $\mathbb{R}^{d}$ satisfying $\lim _{n \rightarrow \infty}\left|x_{n}\right|=\infty$. Recall that $\|u\|_{2}=\left\|u_{n}\right\|_{2}$ for all $n$ and furthermore by the Riesz rearrangement inequality (1.4), we know that

$$
-\mathcal{W}\left(u_{n}, v_{n}\right) \leq-\mathcal{W}(u, v)
$$

Finally, we have that

$$
\lim _{n \rightarrow \infty} \mathcal{S}\left(u_{n}, v_{n}\right)=0
$$

Thus, there exists an $N$ sufficiently large so that $\mathcal{F}\left(u_{N}, v_{N}\right)<\mathcal{F}\left(u^{*}, v^{*}\right)$, which is a contradiction.

REMARK 3.1. Note that for (1.21) to hold it is necessary that $G(x)$ have bounded support.

It is useful to rewrite the energy (1.8) with $\sigma=0$ as

$$
\mathcal{F}[u, v](x)=\mathcal{F}_{1}(u)+\mathcal{F}_{1}(v)+\mathcal{S}(u, v),
$$

where

$$
\mathcal{F}_{1}(w)=\eta \int w^{2} d x-\frac{1}{2} \iint G(x-y) w(x) w(y) d x d y
$$

Let us define $\liminf _{w \in \mathcal{Y}_{M}} \mathcal{F}_{1}[w]:=I_{M}$, where $\mathcal{Y}_{M}=\left\{u \in L^{1} \cap L^{2}:\|u\|_{1}=M\right\}$.

We now state a result which reduces the problem of finding minimizers to (1.8) in $\mathcal{Y}_{M_{u}, M_{v}}$ for $M_{u}, M_{v}>0$ to finding minimizers of (3.9) in $\mathcal{Y}_{M}$ for $M>0$.

LEMMA 3.2 (Reduction of minimizer problem). Let $G$ have compact support, i.e. $\operatorname{supp}(G) \subset B_{R}(0)$ for some $R>0$. 
(i) If the infimum of $\mathcal{F}_{1}$ in $\mathcal{Y}_{M}$ for $M>0$ is achieved by a function with compact support, then there exist a minimizer $(u, v) \in \mathcal{Y}_{M_{u}, M_{v}} \cap C_{0}^{2}(\Omega) \times C_{0}^{2}(\Omega)$ of $\mathcal{F}$. In particular, if $\left(u^{*}, v^{*}\right)$ such that $u^{*} \in \mathcal{Y}_{M_{u}}$ is a minimizer of $\mathcal{F}_{1}(u)$ and $v^{*} \in \mathcal{Y}_{M_{v}}$ is a minimizer of $\mathcal{F}_{1}(v)$ with bounded support then there exists $x_{1} \in \mathbb{R}^{d}$ such that $\left(u^{*}\left(\cdot+x_{1}\right), v^{*}\left(\cdot-x_{1}\right)\right)$ is a minimizer of $\mathcal{F}$ in $\mathcal{Y}_{M_{u}, M_{v}}$.

(ii) If all minimizers of $\mathcal{F}_{1}$ in $\mathcal{Y}_{M}$ for $M>0$ have unbounded support then $\mathcal{F}$ does not achieve its minimum in $\mathcal{Y}_{M_{u}, M_{v}}$ for any $M_{u}, M_{v}>0$.

(iii) Any $\left(u^{*}, v^{*}\right) \in \mathcal{Y}_{M_{u}, M_{v}}$ which minimizes $\mathcal{F}[u, v]$ must minimize $\mathcal{F}_{1}$ individually.

Proof. The proof of (i) is clear. To prove (ii), recall that any minimizer of $\mathcal{F}$ must have compact support, thus we assume for contradiction that there exits a minimizer $(u, v) \in \mathcal{Y}_{M_{1}, M_{2}}$. Since $u$ cannot be a minimizer for $\mathcal{F}_{1}$ then

$$
\mathcal{F}_{1}(u)+\mathcal{F}_{1}(v)>\mathcal{F}_{1}\left(u^{*}\right)+\mathcal{F}_{1}\left(v^{*}\right)
$$

where $\left(u^{*}, v^{*}\right) \in \mathcal{Y}_{M_{u}, M_{v}}$ are both minimizer of $\mathcal{F}_{1}$. Now, define

$$
u_{n}=\left(u^{*}\right)^{\#}\left(\cdot-x_{n}\right) \text { and } v_{n}=\left(v^{*}\right)^{\#}\left(\cdot+x_{n}\right)
$$

for a sequence $\left\{x_{n}\right\} \subset \mathbb{R}^{d}$. Let $N$ be such that $\left|x_{N}\right|$ is sufficiently large with

$$
S\left(u_{N}, v_{N}\right)<\mathcal{F}_{1}(u)+\mathcal{F}_{1}(v)-\mathcal{F}_{1}\left(u^{*}\right)-\mathcal{F}_{1}\left(v^{*}\right) .
$$

From this we conclude that $\mathcal{F}\left(u_{N}, v_{N}\right)<\mathcal{F}(u, v)$ which is a contradiction. The proof of (iii) is the same as that of (ii).

We are now ready to prove Proposition 1.5.

Proof. $\quad$ By Proposition 3.1 in [1] we know that any minimizer $u \in \mathcal{Y}_{m}$ of $\mathcal{F}_{1}$ satisfies

$$
u \nabla \cdot(2 \eta u-G * u)=0 \quad \text { a.e. in } \quad \mathbb{R}^{d} .
$$

This in conjunction with the fact that any minimizer $(u, v) \in \mathcal{Y}_{M_{u}, M_{v}}$ of $\mathcal{F}[u, v]$ must have disjoint support, specifically the support of $u$ and the support of $v$ must be separated by $2 R$ if $\operatorname{supp}(G) \subset B_{R}(0)$. This gives the result.

Proof. (Proposition 1.6.) From Proposition 1.1 with $\sigma=\Gamma_{i}=0$ we obtain that

$$
\mathcal{F}(t)+\int_{0}^{t} \int_{\Omega} u \mid \nabla\left(2 \eta u+\left.G *(v-u)\right|^{2}+v \mid \nabla\left(2 \eta v+\left.G *(u-v)\right|^{2} d x d s \leq \mathcal{F}(0),\right.\right.
$$

for all $t>0$. Thus, we have

$$
\begin{aligned}
& 4 \eta^{2} \int_{\Omega} u|\nabla u|^{2}+v|\nabla v|^{2} d x+4 \eta \int_{\Omega} u \nabla u \nabla G *(u-v) \\
& +v \nabla v \nabla G *(u-v) d x+\int_{\Omega}(u+v)|\nabla G *(u-v)|^{2} d x<\infty,
\end{aligned}
$$

for almost every $t>0$. An application of Cauchy-Schwarz inequality gives (1.20). Additionally, if $(u, v)$ are steady-state solutions then they satisfy

$$
u \mid \nabla\left(2 \eta u+\left.G *(v-u)\right|^{2}+v \mid \nabla\left(2 \eta v+\left.G *(u-v)\right|^{2}=0,\right.\right.
$$

for almost all $x \in \mathbb{R}^{d}$. This implies that

$$
2 \eta u+G *(v-u)=C_{1} \quad \text { and } \quad 2 \eta v+G *(u-v)=C_{2},
$$


for some constants $C_{1}, C_{2}$ almost everywhere on every connected component of the support. By properties of the convolution we have that $u$ and $v$ inherit the regularity of $G$.

Proof. (Theorem 1.8.) For an interaction potential $G(x)$ with unbounded support we follow a similar argument of that given in the proof of Lemma 1.7. Taking the distance between the center of mass of a sequence $u_{n}$ and sequence $v_{n}$ to infinity will decrease the segregation energy to zero and a minimizer will never be achieved. Thus, without loss of generality, let us take $G(x)$ to have compact support with $\|G\|_{1}<2 \eta$. Assume that there exists a minimizer $(u, v) \in \mathcal{Y}_{M_{u}, M_{v}}$, we have the lower bound

$$
\mathcal{F}(u, v) \geq\left(\|u\|_{2}^{2}+\|v\|_{2}^{2}\right)\left(\eta-\frac{\|G\|_{1}}{2}\right)
$$

if $\|G\|<2$ then $\mathcal{F}(u, v)>0$ which is a contradiction as $I_{M_{1}, M_{2}} \leq 0$.

Segregation due to mobility disparity and environment - the local case. In this section, we prove Theorem 1.10, which requires the following lemma.

Lemma 3.1. Let $A(x) \in L^{\infty}(\Omega)$. There are solutions, $\bar{u}(x), \bar{v}(x) \in L^{1}(\Omega)$, to

$$
\begin{aligned}
& u(x)=c_{1} \exp \left\{-\frac{1}{\sigma}\left(\Gamma_{1} A(x)+\lambda_{2} v(x)\right)\right\}, \\
& v(x)=c_{2} \exp \left\{-\frac{1}{\sigma}\left(\Gamma_{2} A(x)+\lambda_{2} u(x)\right)\right\} .
\end{aligned}
$$

In addition, for any $M_{1}, M_{2}>0$ we may choose $c_{1}$ and $c_{2}$ such that

$$
c_{1} \int_{\Omega} \bar{u}(x) d x=M_{1} \quad \text { and } \quad c_{2} \int_{\Omega} \bar{v}(x) d x=M_{2} .
$$

Proof. (Lemma 3.1.) The proof is in two steps: in the first step we prove existence of a fixed point of system (3.11) when $c_{1}$ and $c_{2}$ are arbitrary positive constants and in the second step we prove that two such constants exist so that (3.12) holds.

Step 1: We set $\sigma=1$ and $\lambda_{2}=1$ without loss of generality. For a fixed $\bar{x} \in \Omega$, we set the constants $\tilde{c}_{1}=c_{1} e^{-\Gamma_{1} A(\bar{x})}$ and $\tilde{c}_{2}=c_{2} e^{-\Gamma_{2} A(\bar{x})}$, so that

$$
u(\bar{x})=\tilde{c}_{1} \exp \left\{-\tilde{c}_{2} \exp \{-u(\bar{x})\}\right\} .
$$

Note that given $u(\bar{x})$ we can find $v(\bar{x})$ using (3.11b). First, observe that for $u(\bar{x})=0$ the left-hand side of (3.13) is smaller than the right-hand side. However, for $u(\bar{x})>\tilde{c}_{1}$ the right-hand side is smaller than the left-hand side. Thus, by continuity and the intermediate value theorem, there exists a positive fixed point $\bar{u}(\bar{x})$, and, correspondingly, $\bar{v}(\bar{x})>0$, that satisfy (3.11) for $\bar{x}$. Following this procedure for all $x \in \Omega$, we obtain the existence of functions $\bar{u}(x)$ and $\bar{v}(x)$ which satisfy (3.11) in all of the domain. Moreover, the fixed points can be chosen so that $\bar{u}(x)$ and $\bar{v}(x)$ are continuous.

Step 2: Next, we prove that there exists $c_{1}$ and $c_{2}$ such that (3.12) holds. Note that $\bar{u}(x)$ and $\bar{v}(x)$ which satisfy (3.11) with finite $c_{1}, c_{2}$ are in $L^{\infty}(\Omega)$ and consequently in $L^{1}(\Omega)$. For simplicity, we define

$$
f(x)=e^{-\Gamma_{1} A(x)} \quad \text { and } \quad g(x)=e^{-\Gamma_{2} A(x)} .
$$


As we want $M[\bar{u}(x)]=M_{1}$ and $M[\bar{v}(x)]=M_{2}$ we set

$$
\int_{\Omega} \bar{v}(x) d x=c_{2} \int_{\Omega} g(x) e^{-\bar{u}(x)} d x=M_{2},
$$

which solving for $c_{2}$ gives

$$
c_{2}=\frac{M_{2}}{\int_{\Omega} g(x) e^{-\bar{u}(x)} d x} .
$$

Therefore, given $M_{2}>0$ we may set $c_{2}$ as above, and insert expression (1.17b) into (1.17a). This will give a single equation for the function $\bar{u}(x)$, parametrized by the constant $c_{1}$, and our task is to show that there exists $c_{1}>0$ so that $M[\bar{u}(x)]=M_{1}$. Consider the mass of $\bar{u}(x)$ :

$$
\begin{aligned}
\int_{\Omega} \bar{u}(x) d x & =c_{1} \int_{\Omega} f(x) \exp \left\{-c_{2} g(x) \exp \{-\bar{u}(x)\}\right\} d x \\
& =c_{1} \int_{\Omega} f(x) \exp \left\{-\frac{M_{2}}{\int_{\Omega} g(y) e^{-\bar{u}(y)} d y} g(x) \exp \{-\bar{u}(x)\}\right\} d x
\end{aligned}
$$

where we have used (3.14). Note that if $c_{1}=0$, then $M[\bar{u}(x)]=0$. In addition, we have the following lower bound for the mass of $\bar{u}(x)$

$$
\begin{aligned}
\int_{\Omega} \bar{u}(x) d x & =c_{1} \int_{\Omega} f(x) \exp \left\{-\frac{M_{2}}{\int_{\Omega} g(y) e^{-\bar{u}(y)} d y} g(x) \exp \{-\bar{u}(x)\}\right\} d x \\
& \geq c_{1} e^{-\Gamma_{1} A_{\max }} \exp \left\{-\frac{M_{2} \kappa}{e^{-\Gamma_{2} A_{\max }}}\right\} \mu\left\{x \in \Omega: e^{-\bar{u}(x)} \leq \kappa \int_{\Omega} e^{-\bar{u}(y)} d y\right\}
\end{aligned}
$$

for any $\kappa \geq 1$ and $A_{\max }$ is the maximum value of $A$ in $\Omega$. Now, we have the bound

$$
\mu\left\{x \in \Omega \mid e^{-\bar{u}(x)} \geq \kappa \int_{\Omega} e^{-\bar{u}(y)} d y\right\} \leq \gamma|\Omega|,
$$

for $\kappa \geq 1$ and $\gamma<1$ such that

$$
\kappa \gamma|\Omega|>1
$$

Thus, we have the bound

$$
\int_{\Omega} \bar{u}(x) d x \geq c_{1} e^{-\Gamma_{1} A_{\max }} \exp \left\{-\frac{M_{2} \kappa}{e^{-\Gamma_{2} A_{\max }}}\right\}|\Omega|(1-\gamma) .
$$

Note that $\gamma$ and $\kappa$ only depend on the size of $\Omega$. From (3.18) we conclude that for $c_{1}$ sufficiently large (and depending on $M_{2}$ ) we have $M[\bar{u}(x)]>M_{1}$. Thus, by continuity there exists a $c_{1}$ such that $M[\bar{u}]=M_{1}$ and $c_{2}$ is then determined from (3.14). With this we conclude the proof.

We are now ready to prove Theorem 1.10.

Proof. (Theorem 1.10.) Inspired by the Fokker-Plank type nature of the system (1.6), we rewrite it

$$
\begin{aligned}
& u_{t}=\sigma \nabla \cdot\left(e^{-\frac{1}{\sigma}\left(\Gamma_{1} A(x)+\lambda_{2} v(x)\right)} \nabla \cdot\left(e^{\frac{1}{\sigma}\left(\Gamma_{1} A(x)+\lambda_{2} v(x)\right)} u\right)\right), \\
& v_{t}=\sigma \nabla \cdot\left(e^{-\frac{1}{\sigma}\left(\Gamma_{2} A(x)+\lambda_{2} u(x)\right)} \nabla \cdot\left(e^{\frac{1}{\sigma}\left(\Gamma_{2} A(x)+\lambda_{2} u(x)\right)} v\right)\right),
\end{aligned}
$$


with the initial conditions $u(x, 0)=u_{0}(x)$ and $v(x, 0)=v_{0}(x)$ and no-flux boundary conditions. From (3.19), we observe that $\bar{u}(x)$ and $\bar{v}(x)$, satisfying (3.11), are steady-state solutions to (1.6) for any $c_{1}, c_{2}>0$ (setting $\sigma=\lambda_{2}=1$ ). Therefore, we are left to verify that they satisfy the no-flux boundary conditions (1.6d) and (1.6e) and the mass property (1.22c). Taking the gradient of $\bar{u}(x)$ and $\bar{v}(x)$ from (3.11) we observe that

$$
\begin{aligned}
& \sigma \nabla \bar{u}(x)=\bar{u}(x) \nabla\left(-\Gamma_{1} A(x)+\lambda_{2} \bar{v}(x)\right), \\
& \sigma \nabla \bar{v}(x)=\bar{v}(x) \nabla\left(-\Gamma_{2} A(x)+\lambda_{2} \bar{u}(x)\right),
\end{aligned}
$$

guaranteeing that the no-flux boundary conditions are satisfied for $\bar{u}(x)$ and $\bar{v}(x)$. Finally, from Lemma 3.1 we can set $M_{1}=M\left[u_{0}(x)\right]$ and $M_{2}=M\left[v_{0}(x)\right]$ and with this we conclude.

\section{Continuous income spectrum-the macroscopic equations}

In this section, we consider the case where the mobility of individuals is continuous, corresponding to a continuous spectrum of incomes in the population. The dynamics of individuals is governed similarly to the case of discrete mobility with some modifications. First, since the mobility is continuous, an individual will be attracted to another individual if the difference in their mobility (representing the difference in economic status) is within the mobility threshold, $\kappa$, (see Section 1.1.3) and they are repulsed otherwise. This brings about a second change, which is that everyone has a short-range repulsion from everyone else regardless of their mobility.

We begin with $n$ different groups, each with $N_{i}$ agents, for $i=1, \ldots, n$, which exhibit intra-group and inter-group interactions. Every member of group $i$ has mobility $y_{i} \in$ $[0,1]$. Moreover, we assume for simplicity that $y_{i}=\frac{i}{n}$ so that the mobility is uniformly distributed. As in the two-population case, we denote by $x_{N_{i}}^{k}(t) \in \mathbb{R}^{d}$ the spatial position of the $k$-th individual in group $i$ which has $N_{i}$ members. The short-range repulsion dynamics is governed by the potential $V_{N}(x)$ and the mobility segregation is governed by the potential $H(y) G_{N}(x)$. Recall that $H\left(y_{i}-y_{j}\right)$ measures the difference in mobility between individuals in groups $i$ and $j$. The sign of $H$ determines whether the individuals are attracted or repulsed from each other. The interaction potential $G_{N}(x)$ takes the physical positioning of the two individuals into account and determines the strength and direction of the interaction. Finally, individuals are advected by the velocity field, $\nabla_{x} A(x)$, with the speed proportional to the mobility.

Combining the above interactions yields the system of evolution equations

$$
\begin{aligned}
\frac{d x_{N_{i}}^{k}(t)}{d t}= & -\frac{1}{n} \sum_{j=1}^{n} \frac{1}{N} \sum_{l=1}^{N} \nabla_{x}\left[V_{N}\left(x_{N_{i}}^{k}(t)-x_{N_{j}}^{l}(t)\right)+H\left(y_{i}-y_{j}\right) G_{N}\left(x_{N_{i}}^{k}(t)-x_{N_{j}}^{l}(t)\right)\right] \\
& -y_{i} \nabla_{x} A\left(x_{N_{i}}^{k}(t)\right),
\end{aligned}
$$

for $k=1, \ldots, N_{i}$, and $i=1, \ldots, n$. We let the number of particles in each group be the same, $N_{i}=N$, for all $i=1, \ldots, n$ (although we continue to use $N_{i}$ to differentiate intergroup interactions). Let $Y_{N_{i}}^{k}(t):=\left(x_{N_{i}}^{k}(t), y_{i}\right)$ and define the empirical measure

$$
Y_{N, n}(t)=\frac{1}{N n} \sum_{i=1}^{n} \sum_{k=1}^{N} \delta_{Y_{N_{i}}^{k}(t)} .
$$

Assume that there are functions $u(x, y, t) \in \mathbb{R}^{d} \times[0,1] \times[0, \infty)$ such that for all $f \in$ $C_{0}^{2}\left(\mathbb{R}^{d+1}\right)$

$$
\left\langle Y_{n N}(t), f\right\rangle \rightarrow\langle u(x, y, t), f\rangle \text { as } n, N \rightarrow \infty
$$


Define

$$
g_{N, n}(x, y, t):=\frac{1}{n N} \sum_{j=1}^{n} \sum_{l=1}^{N} \nabla_{x}\left(V_{N}\left(x-x_{N_{j}}^{l}(t)\right)+H\left(y-y_{j}\right) G_{N}\left(x-x_{N_{j}}^{l}(t)\right)-y A(x)\right),
$$

then (4.1) can be written as

$$
\frac{d x_{N_{i}}^{k}(t)}{d t}=-\nabla_{x} g_{N, n}\left(x, \frac{i}{n}, t\right)
$$

for $k=1, \ldots, N$ and $i=1, \ldots, n$. Let $f \in C_{0}^{2}\left(\mathbb{R}^{d+1}\right)$ then for $N$ and $n$ finite we have

$$
\left\langle Y_{N, n}(t), f(x, y)\right\rangle=\frac{1}{n N} \sum_{i=1}^{n} \sum_{k=1}^{N} f\left(x_{N_{i}}^{k}(t), \frac{i}{n}\right) .
$$

Taking the time derivative of (4.4), substituting in the evolution equation (4.3), and integrating gives

$$
\left\langle Y_{N, n}(t), f(x, y)\right\rangle=\left\langle Y_{N, n}(0), f(x, y)\right\rangle+\int_{0}^{t}\left\langle Y_{N, n}(s),-\nabla_{x} g_{N, n} \cdot \nabla_{x} f\right\rangle d s .
$$

Consider for simplicity the one-dimensional case, and denote $h^{\prime}=\partial_{x} h$. Then we may write

$$
\partial_{x} g_{N, n}(x, y, t)=\frac{1}{n N} \sum_{j=1}^{n} \sum_{l=1}^{N}\left[V_{N}^{\prime}\left(x-x_{N_{j}}^{l}(t)\right)-H\left(y-\frac{j}{n}\right) G_{N}^{\prime}\left(x-x_{N_{j}}^{l}(t)\right)\right]+y A^{\prime}(x) .
$$

The only problematic term in (4.5) is the integral in time, which in one dimension is

$$
\begin{aligned}
\left\langle Y_{N, n}(t),-\nabla_{x} g_{N, n} \cdot \nabla_{x} f\right\rangle= & -\frac{1}{(n N)^{2}} \sum_{i, j=1}^{n} \sum_{k, l=1}^{N} f^{\prime}\left(x_{N_{i}}^{k}(t)\right) V_{N}^{\prime}\left(x_{N_{i}}^{k}(t)-x_{N_{j}}^{l}(t)\right) \\
& -\frac{1}{(n N)^{2}} \sum_{i, j=1}^{n} \sum_{k, l=1}^{N} f^{\prime}\left(x_{N_{i}}^{k}(t)\right) H\left(\frac{i}{n}-\frac{j}{n}\right) G_{N}^{\prime}\left(x_{N_{i}}^{k}(t)-x_{N_{j}}^{l}(t)\right) \\
& +\frac{1}{n N} \sum_{i=1}^{n} \sum_{k=1}^{N} y_{i} A^{\prime}\left(x_{N_{i}}^{k}(t)\right) f^{\prime}\left(x_{N_{i}}^{k}(t)\right):=I_{1}+I_{2}+I_{3} .
\end{aligned}
$$

Since we are assuming short-range repulsion and long-range mobility segregation we take $\gamma_{v} \in(0,1)$ and $\gamma_{g}=0$. Consider an individual $x$ and their interactions with individuals from group $i$ (which has mobility $y_{i}$ ). Due to the local equilibrium hypothesis, we expect that as $N \rightarrow \infty$ the distances between individuals near $x$ in group $i$ at time $t$ are approximately

$$
\frac{1}{N u\left(x, y_{i}, t\right)}
$$

Using this in (4.7), we obtain

$$
\begin{aligned}
I_{2} & =-\frac{1}{(n N)^{2}} \sum_{i, k=1}^{n} \sum_{j, l=1}^{N} f^{\prime}\left(x_{N_{i}}^{j}(t), y_{i}\right) H\left(y_{i}-y_{k}\right) G_{N^{\prime}}^{\prime}\left(x_{N_{i}}^{j}(t)-x_{N_{k}}^{l}(t)\right) \\
& =\left\langle Y_{n N}(t), f^{\prime}(\cdot) \frac{1}{n N} \sum_{k=1}^{n} \sum_{l=1}^{N} H\left(\cdot-y_{k}\right) G_{N}\left(\cdot-x_{N_{k}}^{l}(t)\right)\right\rangle .
\end{aligned}
$$


Now, assuming that $G_{N}^{\prime}(x)$ decays sufficiently fast as $|x| \rightarrow \infty$, by (4.7) we can approximate the position of the individuals of group $k$ by $l / N u\left(x_{l}, \frac{k}{n}, t\right)$ for $l=-N / 2, \ldots, N / 2$. This gives the approximation

$$
\frac{1}{n N} \sum_{k=1}^{n} \sum_{l=-N / 2}^{N / 2} H\left(\cdot-y_{k}\right) G_{N}\left(\cdot-x_{N_{k}}^{l}(t)\right) \approx \int_{0}^{1} \int_{\mathbb{R}} H(\cdot-\tilde{y}) G^{\prime}(\cdot-\tilde{x}) u(\tilde{x}, \tilde{y}, t) d \tilde{x} d \tilde{y} .
$$

For $I_{1}$, by symmetrization and the Taylor expansion of $f^{\prime}$, we obtain

$$
\begin{aligned}
I_{1} & =\frac{1}{2(n N)^{2}} \sum_{i, j=1}^{n} \sum_{k, l=1}^{N}\left[f^{\prime}\left(x_{N_{i}}^{k}(t)\right)-f^{\prime}\left(X_{N_{j}}^{l}(t)\right)\right] V_{N}^{\prime}\left(x_{N_{i}}^{k}(t)-x_{N_{j}}^{l}(t)\right) \\
& =\left\langle Y_{N, n}(t), f^{\prime \prime} \frac{1}{2(n N)} \sum_{j=1}^{n} \sum_{l=1}^{N} V_{N}^{\prime}\left(x_{N_{i}}^{k}(t)-x_{N_{j}}^{l}(t)\right)\left(x_{N_{i}}^{k}(t)-x_{N_{j}}^{l}(t)\right)\right\rangle .
\end{aligned}
$$

By the local equilibrium hypothesis we have $x_{N_{i}}^{k}(t)-x_{N_{j}}^{l}(t) \approx \frac{l}{N u\left(\cdot, \frac{k}{n}, t\right)}$. Using $(2.1)$ and denoting $V_{1}(x)=V(x)$, we obtain

$$
\begin{aligned}
\frac{1}{n N} \sum_{k=1}^{n} \sum_{l=1}^{N} V_{n N}^{\prime}\left(\cdot-x_{N_{k}}^{l}(t)\right)\left(\cdot-x_{N_{k}}^{l}\right) & \approx \frac{1}{n} \sum_{k=1}^{N}\left(\frac{N^{\gamma_{v}}}{N} \sum_{l=-N / 2}^{N / 2} V^{\prime}\left(\frac{N^{\gamma_{v}} l}{N u\left(\cdot, \frac{k}{n}, t\right)}\right) \frac{N^{\gamma_{v}} l}{N u\left(\cdot, \frac{k}{n}, t\right)}\right) \\
& \approx-M[V] \int_{0}^{1} u(\cdot, y, t) d y .
\end{aligned}
$$

Here, we approximated the Riemann sum by an integral. Recall that $u(x, t)$ defined in (1.25) measures the population density at location $x$ and time $t$ over all mobilities. The term that is contributed by the environment, $I_{3}$, is derived similarly and we omit these steps. Combining the above calculations gives the weak version of (1.24).

\section{Numerical results}

Numerical simulations for the interacting-particle model. We performed numerical simulations of the interacting-particle system with multiple groups of varying economic status. Figure 5.1a illustrates the equilibrium state when only the dynamic rules (A1) and (A2) are considered. We observe a degree of segregation but some social diversity is preserved. On the other hand, the inclusion of the effects of a monocentric environment, where all resources are concentrated in the city center, leads to an exaggerated segregation state. Individuals with a higher mobility live in the center of the city while the other groups form regions surrounding the city center with increasing distance to the city center as the mobility decreases.

Numerical solutions to the local continuous limit. Figure 5.3 illustrates numerical solutions to (1.6) where the environment, $A(x)$, represents a city with two main regions where all amenities are located, see Figure 5.2a. Initially, both populations (disadvantaged individuals and affluent individuals occupy the same space) - this is illustrated in Figure 5.2b and Figure 5.2c. In Figure 5.3a and Figure 5.3b we observe the steady-state solutions for $u(x, t)$ and $v(x, t)$, respectively, when there is no mobility or environmental influence. We observe that due to the social preference $u(x, t)$ and $v(x, t)$ occupy different spaces. On the other hand when we include mobility and the environment we observe that $u(x, t)$ occupies the space, where $A(x)$ is large and $v(x, t)$ occupies the rest of the domain - see Figures 5.3c and 5.3d. Finally, Figures 5.3a 


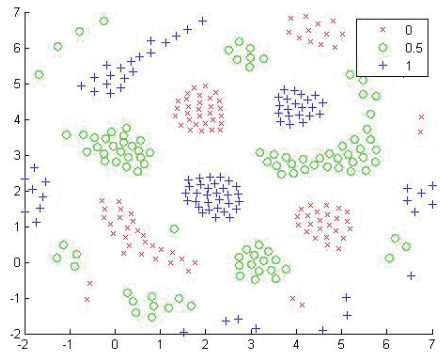

(a) $\Gamma_{1}=\Gamma_{2}=\Gamma_{3}=0$

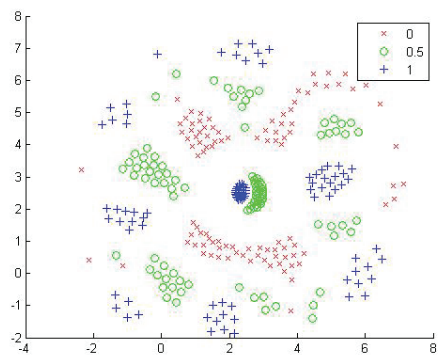

(c) $\Gamma_{1}=5, \Gamma_{2}=2.5, \Gamma_{3}=0$

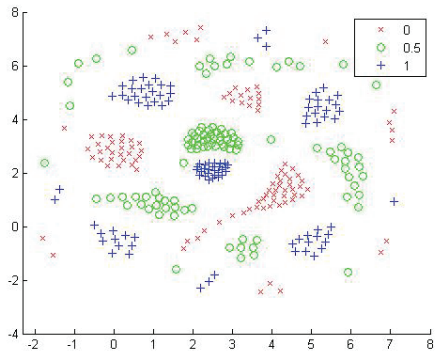

(b) $\Gamma_{1}=1, \Gamma_{2}=.5, \Gamma_{3}=0$

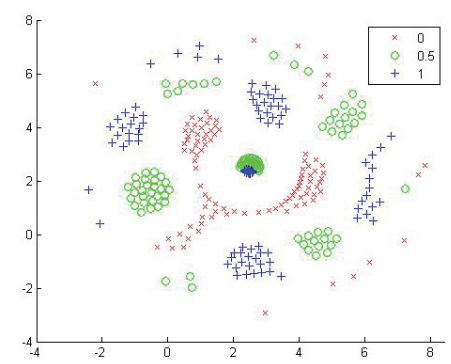

(d) $\Gamma_{1}=10, \Gamma_{2}=5, \Gamma_{3}=0$

Fig. 5.1: Results of numerical simulations of the interacting particle system with three sub-populations with mobilities, $\Gamma_{1}, \Gamma_{2}$, and $\Gamma_{3}$, and an initial random distribution. Figure 5.1 a illustrates the equilibrium distribution when only social preference is taken into account. Figures 5.1b, 5.1c, and 5.1d illustrates the equilibrium state when both the environment and economic disparity are taken into account with increasing mobility gap.

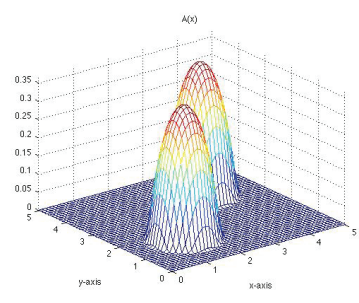

(a) $\mathrm{A}(\mathrm{x})$

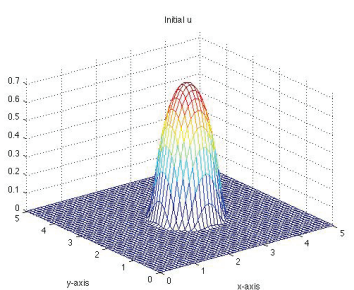

(b) $\mathrm{u}(\mathrm{x}, 0)$

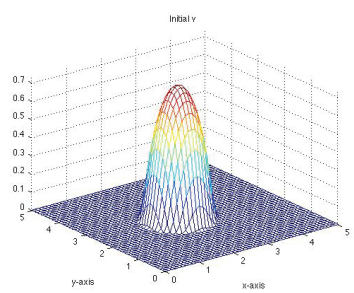

(c) $\mathrm{v}(\mathrm{x}, 0)$

Fig. 5.2

and $5.3 \mathrm{~b}$ illustrate the case when the social preference is zero and we see that $v(x, t)$ disperses.

Crime, safety, and economic disparity As mentioned in the introduction it is well known that poor neighborhoods have a disproportionate amount of crime [13,14]. In this section we briefly describe how one can explore the effect of segregation along income lines on crime hotspots using a mathematical model. The results yield distributions of crime that are qualitatively similar to empirical observations of crime density. We 


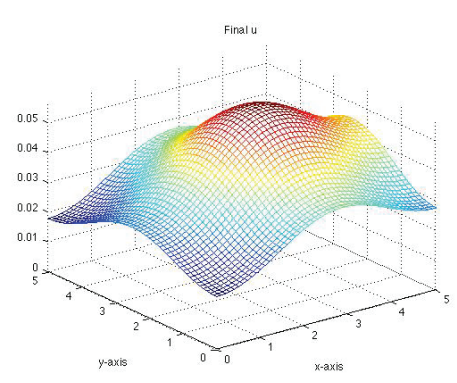

(a) $\lambda_{2}=1, \Gamma_{1}=\Gamma_{2}=0: u$

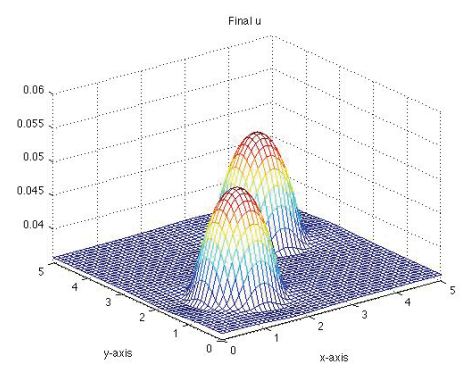

(c) $\lambda_{2}=1, \Gamma_{1}=1, \Gamma_{2}=0: u$

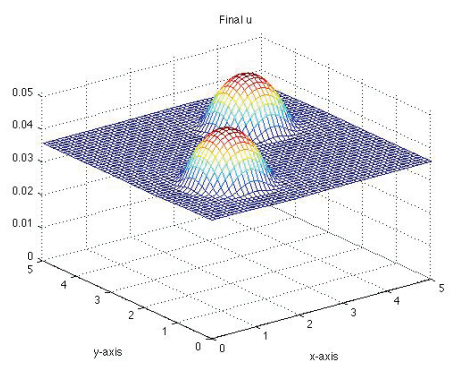

(a) $\lambda_{2}=0, \Gamma_{1}=1, \Gamma_{2}=0: u$

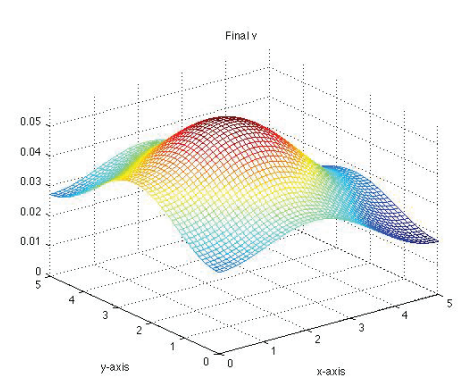

(b) $\lambda_{2}=1, \Gamma_{1}=\Gamma_{2}=0: v$

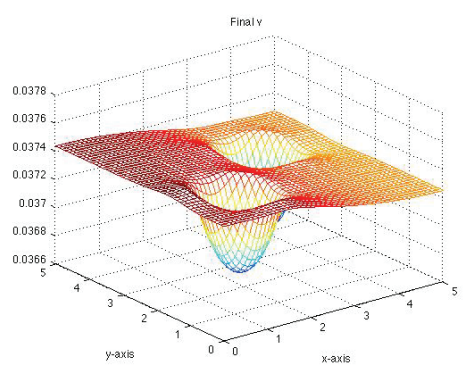

(d) $\lambda_{2}=1, \Gamma_{1}=1, \Gamma_{2}=0: v$

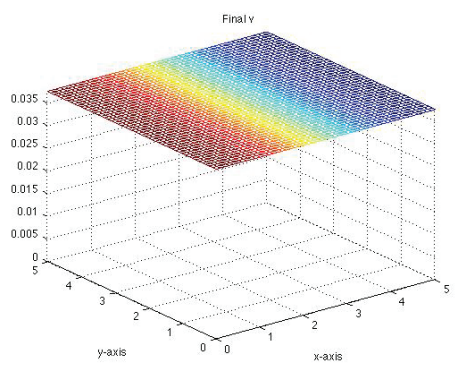

(b) $\lambda_{2}=0, \Gamma_{1}=1, \Gamma_{2}=0: v$

Fig. 5.3: Numerical solutions to (1.6) with the heterogeneous environment illustrated in Figure 5.2a and initial conditions for $u(x, t)$ and $v(x, t)$ illustrated in Figures 5.2b and 5.2c respectively with varying $\lambda_{2}$ and $\Gamma_{1}\left(\Gamma_{2}=0\right)$.

summarize the assumptions for this model:

(i) The police enforcement aims to minimize feelings of insecurity.

(ii) There are limited resources.

(iii) Insecurity is higher among people that have more resources and have a lower tolerance for criminal activity.

Given a distribution of individuals with prescribed mobilities, we calculate the influence field, $I(x)$, by adding delta functions which are are centered at every location 
where an individual lives and with a mass that depends on the mobility of the individual. Once we obtain the influence field, the objective is to find the distribution of the police resources, $p(x)$, that minimizes the functional (1.27), as described in (1.28). This is done numerically with the use a gradient descent method, which requires a soft version of the constrain (1.28b). Thus, we minimize

$$
F(t)=\int_{\Omega} \epsilon\left(|\nabla p(x)|^{2}+U(p(x))\right) d x+\frac{1}{\epsilon}\left(\int_{\Omega} p(x) d x-1\right)^{2},
$$

for $\epsilon \ll 1$, which gives more weight to the terms that requires the constraint on the total amount of the police enforcement to be satisfied.

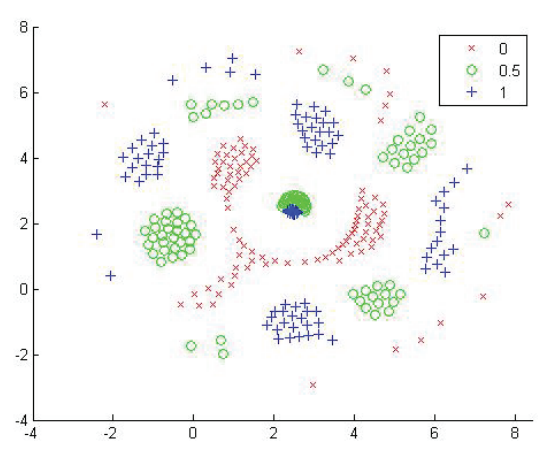

(a) Distribution of the population

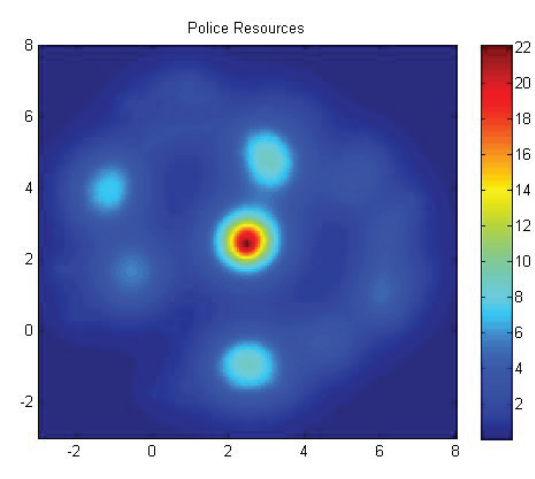

(b) Distribution of the police resources

Fig. 5.4

Figure 5.4a illustrates the distribution of the population from which we generate an influence field, $I(x)$. Figure 5.4b illustrates the optimal distribution of resources obtained based on the given $I(x)$. We observe the development of safe-havens for regions with a high influence field, where the crime fields are very low. While this does not generate the crime hotspots, it essentially removes the possibility of hotspots in regions of high influence. The next step, which we leave for a separate publication is to couple the environment attractiveness field, $A(x)$, we have considered in the PDE models, to the amount of crime (which would be described via the local police resources) produced via the above minimization procedure. This will lead to a coupled system of PDEs for the population density and the police enforcement.

Acknowledgments. We are grateful to Georg Menz for numerous helpful discussions, and to Henri Berestycki and Jean-Pierre Nadal for their interest in this work and encouragement. NR was partially supported by the NSF Postdoctoral Fellowship in Mathematical Sciences DMS-1103765, and LR by an NSF grant DMS-1311903.

\section{REFERENCES}

[1] M. Burger, M. Di Francesco, and M. Franek, Stationary states of quadratic diffusion equations with long-range attraction, AxXiv ID: 1103.5365, 1-28, 2012.

[2] S. Bowles and S.-H. Hwang, Social preferences and public economics: Mechanism design when social preferences depend on incentives, Journal of Public Economics, 92(8-9), 1811-1820, August 2008. 
[3] G.S. Becker and K.M. Murphy, Social Economics: Market Behavior in a Social Environment, Harvard University Press/Belknap, Cambridge, 2000.

[4] J. Bedrossian, N. Rodríguez, and A.L. Bertozzi, Local and global well-posedness for aggregation equations and Patlak-Keller-Segel models with degenerate diffusion, Nonlinearity, 24(6), 1-31, 2011.

[5] J. Boston, L.C. Rigsby, and M.N. Zald, The impact of race on housing markets: a critical review, Social Problems, 19(3), 382-393, 1972.

[6] F.A.C.C. Chalub, P. Markowich, B. Perthame, and C. Schmeiser, Kinetic models for chemotaxis and their drift-diffusion limits, Monatshefte für Mathematik, 142(1-2), 123-141, 2004.

[7] M. Di Francesco and S. Fagioli, Measure solutions for nonlocal interaction PDEs with two species, Nonlinearity, 26, 2777-2808, 2013.

[8] M.J. Fischer, The relative importance of income and race in determining residential outcomes in U.S. urban areas, 1970-2000, Urban Affairs Review, 38(5), 669-696, 2003.

[9] D.N. Figlio and M.E. Lucas, What's in a grade? School report cards and house prices, American Economic Review, 94(3), 591-604, 2004.

[10] H. Goldstein and P. Noden, Modelling social segregation, Oxford Review of Education, 29(2), 225-237, June 2003.

[11] L. Gauvin, A. Vignes, and J-P. Nadal, Modeling urban housing market dynamics: can the sociospatial segregation preserve some social diversity? Journal of Economic Dynamics and Control, $37,1300-1321,2013$

[12] Y.M. Ioannides and J.E. Zabel, Neighborhood effects and housing, Journal of Applied Econometrics, 18(5), 563-583, 2003.

[13] L.J. Krivo and R.D. Peterson, Extremeley disadvantaged neighborhoods and urban crime, Social Forces, 75(2), 619-648, 1996.

[14] S.D. Levitt, The changing relationship between income and crime victimization, Economic Policy Review, 5(3), 87-98, 1999

[15] E.H. Lieb, Sharp constants in the Hardy-Littlewood-Sobolev and related inequalities, The Annals of Mathematics, Second Series, 118(2), 349-374, 1983.

[16] E.F.P. Luttmer, Neighbors as negatives: Relative earnings and well-being, The Quarterly Journal of Economics, 120(3), 963-1002, 2005.

[17] G. D. Mahan, Many-particle Physics, Plenum Publishers, New York, 2000.

[18] D. Morale, V. Capasso, and K. Oelschläger, An interacting particle system modelling aggregation behavior: from individuals to populations, Journal of Mathematical Biology, 50(1), 49-66, January 2004.

[19] A. Murie and S. Musterd, Social segregation, housing tenure and social change in Dutch cities in the Late 1980s, Urban Studies, 33(3), 495-516, April 1996.

[20] K. Oelschläger, A law of large numbers for moderately interacting diffusion processes, $\mathrm{Z}$. Wahrscheinlichkeitstheorie verw. Gebiete, 69, 279-322, 1985.

[21] K. Oelschläger, Large systems of interacting particles and the porous medium equation, Journal of Differential Equations, 88, 294-346, 1990.

[22] C. Pantazis, 'Fear of Crime,' vulnerability and poverty: evidence from the British crime survey, Brit. J. Criminol., 40, 414-436, 2000.

[23] S. Sassi, Cultural differentiation or social segregation? Four approaches to the digital divide, New Media \& Society, 7(5), 684-700, October 2005.

[24] T.C. Schelling, Dynamic models of segregation, Journal of Mathematical Sociology, 1, 143-186, 1971.

[25] M. Schienbein, K. Franke, and H. Gruler, Random walk and directed movement: comparison between inert particles and self-organized molecular machines Physical Review E, 49(6), 54625472, 1994.

[26] Y. Seo and R.A. Simons, The effect of school quality on residential sales price, Journal of Real Estate Research, 31(3), 307-327, 2009.

[27] E.T. van Kempen, The dual city and the poor: social polarisation, social segregation and life chances, Urban Studies, 31(7), 995-1015, 1994. 